\title{
Monotone and Conservative Cascade Remapping between Spherical Grids (CaRS): Regular Latitude-Longitude and Cubed-Sphere Grids
}

\author{
Peter H. LAuritzen \\ Climate and Global Dynamics Division, National Center for Atmospheric Research,* Boulder, Colorado \\ RAMACHANDRAN D. NAIR \\ Institute for Mathematics Applied to Geosciences, National Center for Atmospheric Research,* Boulder, Colorado
}

(Manuscript received 20 February 2007, in final form 3 July 2007)

\begin{abstract}
A high-order monotone and conservative cascade remapping algorithm between spherical grids (CaRS) is developed. This algorithm is specifically designed to remap between the cubed-sphere and regular latitude-longitude grids. The remapping approach is based on the conservative cascade method in which a two-dimensional remapping problem is split into two one-dimensional problems. This allows for easy implementation of high-order subgrid-cell reconstructions as well as the application of advanced monotone filters. The accuracy of CaRS is assessed by remapping analytic fields from the regular latitude-longitude grid to the cubed-sphere grid. In terms of standard error measures, CaRS is found to be competitive relative to an existing algorithm when regridding from a fine to a coarse grid and more accurate when regridding from a coarse to a fine grid.
\end{abstract}

\section{Introduction}

The choice of a spherical grid system is crucial for designing efficient scalable global atmospheric models that can exploit the enormous computing potentials of present-day distributed memory parallel machines. Because of the problems associated with the polar grid singularities, the regular latitude-longitude (RLL; see Table 1 for a complete list of acronyms used in this paper) spherical grid is not well suited for being a candidate for developing such scalable models. In recent years, there has been great interest in developing numerical models based on more isotropic spherical grid systems that are free from singularities or contain weaker singularities and are suitable for local numerical methods such as finite-volume or high-order Galerkin methods. For example, the cubed-sphere (spherical

*The National Center for Atmospheric Research is sponsored by the National Science Foundation.

Corresponding author address: Peter Hjort Lauritzen, Climate Modeling Section, Climate and Global Dynamics Division, National Center for Atmospheric Research, 1850 Table Mesa Drive, Boulder, CO 80305.

E-mail: pel@ucar.edu cube or expanded cube) geometry introduced by Sadourny (1972) in the early days of atmospheric modeling has recently been reintroduced by Ronchi et al. (1996) and Rančić et al. (1996) with additional desirable features such as the equiangular grid spacing and orthogonality. This grid system is being considered by many research groups for their new-generation highorder spectral element or finite-volume models (see, e.g., Thomas and Loft 2002; Giraldo and Rosmond 2004; Adcroft et al. 2004; Baer et al. 2006).

General modeling environments are composed of various model components often defined on different grid systems. For example, the atmospheric component may be defined on a cubed-sphere grid whereas the land surface and other components are defined on the RLL grid. For the coupling between model components data must be regridded. There are many practical issues for transferring data from a grid system to another with fidelity. High-order accurate interpolation of field variables from one spherical grid to another without violating properties such as conservation and monotonicity (nonoscillatory) is a daunting task.

Our focus in this paper is the development of a highorder conservative and monotone remapping scheme that is specifically designed to remap between the cubed-sphere class of grid systems and conventional 
TABLE 1. List of acronyms used in this paper.

\begin{tabular}{ll}
\hline \hline & \multicolumn{1}{c}{ Acronym } \\
\hline CaRS & Cascade remapping between spherical grids \\
()-M & Suffix stands for monotone filter applied \\
PCM & Piecewise cubic method \\
PCoM & Piecewise constant method \\
PLM & Piecewise linear method \\
PPM & Piecewise parabolic method \\
PSM & Piecewise spline method \\
RLL & Regular latitude-longitude \\
SCRIP & Spherical Coordinate Remapping and Interpolation \\
& Package \\
\hline
\end{tabular}

latitude-longitude spherical grids. The proposed method could be used for coupling RLL-based physics packages and other model components to cubed-sphere dynamical cores. Moreover this remapping may be employed for pre- and postprocessing of data on the cubed sphere and data visualization on RLL-based graphic packages.

Jones (1999) developed a conservative remapping procedure on the sphere [Spherical Coordinate Remapping and Interpolation Package (SCRIP)] by employing the divergence theorem. This method has great geometric flexibility and is capable of handling different types of spherical grids. The resulting conservative remapping algorithm is up to second-order accurate. For the second-order SCRIP remapping the gradients in spherical coordinates must be approximated and supplied to SCRIP. In a coupled model setting one may use the internal representation of the model numerics, such as spectral methods, finite elements, etc., for the approximation of the gradients.

A new conservative remapping method between the cubed-sphere grids and the RLL grids [hereafter referred to as CaRS (cascade remapping between spherical grids)] is presented. This approach is based on the conservative cascade interpolation framework; therefore, the order of the interpolation (remapping) can relatively easily be varied and there are efficient options for enforcing monotonicity. We compare our results against SCRIP for a set of scalar fields. The remainder of the paper is organized as follows. Section 2 provides a brief review of conservative cascade remapping, and section 3 details the remapping algorithm CaRS between cubed-sphere and RLL grids. Numerical experiments and test results are given in section 4 followed by summary and conclusions in section 5 .

\section{Conservative cascade remapping}

Cascade interpolation was introduced by Purser and Leslie (1991) in the context of semi-Lagrangian advec- tion. Cascade schemes significantly reduce the computational cost of the upstream (or downstream) multidimensional interpolation problem associated with semi-Lagrangian advection by employing an efficient sequence of one-dimensional interpolations. Nair et al. (1999) extended this method to spherical geometry, and a conservative version of the cascade scheme on the sphere was introduced in Nair et al. (2002) and in Zerroukat et al. (2005). In two dimensions, the cascade interpolation method requires the underlying surfaces represented by two families of structured grid lines forming a rectangular mesh.

\section{a. Source and target grids}

For the two-dimensional forward-trajectory semiLagrangian scheme on the sphere (Nair et al. 2003) the source grid is defined by the flow-distorted Lagrangian grid and the target grid is the RLL (Eulerian) grid, but for the popularly used backward-trajectory semiLagrangian scheme the source and target grids are reversed. In either case the cascade interpolation transfers data from the source to target grid through an intermediate grid system by employing a sequence of one-dimensional interpolations (or remappings). Unlike the cascade semi-Lagrangian schemes, the current remapping problem between the source and target grids is not constrained by trajectories. For the CaRS algorithm the source and target grids can be switched between RLL and cubed-sphere grids.

In general the cubed-sphere provides different classes of grid systems such as equiangular, equidistant, and orthogonal (conformal cubic grids) with different spatial properties (for details see Nair et al. 2005). Without loss of generality we consider the equiangular cubed sphere, where a sphere is decomposed into six identical regions (panels or faces) and the grid lines on each panel on the sphere are defined by the equispaced central angles $(\alpha, \beta) \in[-\pi / 4, \pi / 4]$. The panel indexing is shown in Fig. 1. This grid system is nonorthogonal and the resulting curvilinear coordinates are discontinuous at the edges of the panels. Throughout this paper the term cubed sphere refers to this grid system.

\section{b. Basic one-dimensional remapping scheme}

The cascade method relies on dimension splitting, and the one-dimensional remapping scheme is the basic component of the multidimensional cascade algorithm. Consider a one-dimensional domain $I \equiv\left[I_{L}, I_{R}\right]$ spanned by two monotonic grid lines, $\xi_{k}^{(s)}$ and $\xi_{l}^{(t)}$, representing source and target grids, respectively, and with corresponding grid spacing $\delta \xi_{k}^{(s)}=\xi_{k+1}^{(s)}-\xi_{k}^{(s)}, \delta \xi_{l}^{(t)}=$ $\xi_{l+1}^{(t)}-\xi_{l}^{(t)}$. Let $\psi(\xi)$ be a generic density function for 


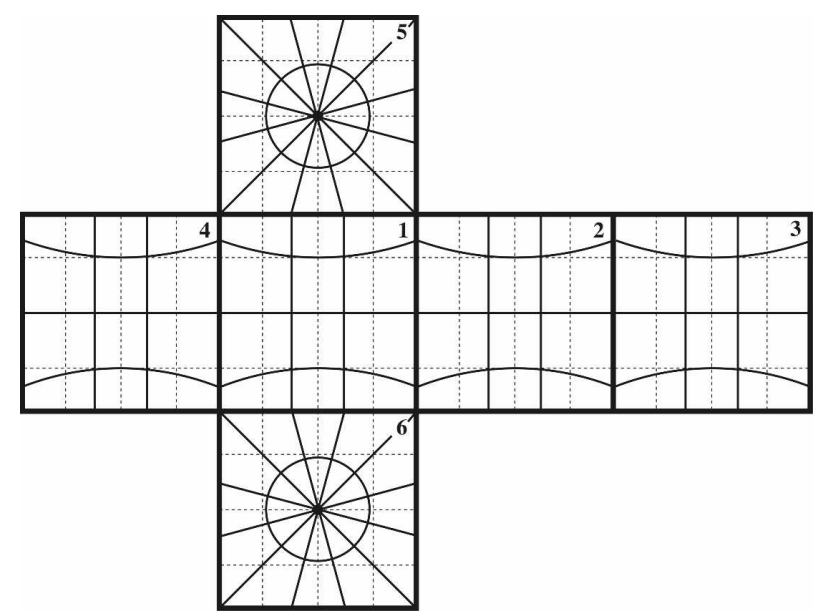

FIG. 1. A schematic illustration of the regular latitude-longitude grid (thin solid lines) on the gnomonic projection. Dashed lines are the cubed-sphere grid lines, and the thick lines mark the border of each panel. The panel index is at the upper-right corner of each panel.

$\xi \in I$. By remapping we transfer the average values $\bar{\psi}\left[\xi^{(s)}\right]$ defined on the source grid to $\bar{\psi}\left[\xi^{(t)}\right]$ on the target grid with mass conservation as a constraint. Also, for consistency it is required that

$$
\left|I_{R}-I_{L}\right|=\sum_{k} \delta \xi_{k}^{(s)}=\sum_{l} \delta \xi_{l}^{(t)} .
$$

The cell-averaged masses in the source grid cells for $\xi \in$ $\left[\xi_{k}^{(s)}, \xi_{k+1}^{(s)}\right]$ are defined by

$$
\bar{\psi}_{k}\left(\xi^{(s)}\right)=\frac{1}{\delta \xi_{k}^{(s)}} \int_{\xi_{k}^{(s)}}^{\xi_{k+1}^{(s)}} \psi(\xi) d \xi .
$$

We follow the "accumulated mass" approach as used in many finite-volume-based remapping schemes (Dukowicz 1984; Rančić 1995; Nair and Machenhauer 2002); that is, the mass in a target grid cell for $\xi \in\left[\xi_{l+1}^{(t)}\right.$, $\left.\xi_{l}^{(t)}\right]$ is given by

$$
\begin{aligned}
\bar{\psi}_{l}\left[\xi^{(t)}\right] \delta \xi_{l}^{(t)} & =\int_{\xi_{l}^{(t)}}^{\xi_{l+1}^{(t)}} \psi(\xi) d \xi \\
& =\int_{I_{L}}^{\xi_{l+1}^{(t)}} \psi(\xi) d \xi-\int_{I_{L}}^{\xi_{l}^{(t)}} \psi(\xi) d \xi .
\end{aligned}
$$

The above equation shows that the masses in the target cells can be represented as the differences of the accumulated mass from a common reference point $I_{L}$. Moreover, the accumulated mass can be expressed in terms of the source-grid variables as follows:

$$
\int_{I_{L}}^{\xi_{l}^{(t)}} \psi(\xi) d \xi=\sum_{n=1}^{q} \bar{\psi}_{n}\left[\xi^{(s)}\right] \delta \xi_{n}^{(s)}+\int_{\xi_{q}^{(s)}}^{\xi_{l}^{(t)}} \psi(\xi) d \xi,
$$

where $\xi_{q}^{(s)} \in\left[\xi_{l-1}^{(t)}, \xi_{l}^{(t)}\right]$ and $\xi_{l}^{s}=I_{L}$. The cell-averaged density field $\bar{\psi}_{l}\left[\xi^{(t)}\right]$ at any target cell can be determined from (3) by employing (4). A schematic illustration of this one-dimensional remapping is shown in Nair and Machenhauer (2002).

In (3) and (4) $\psi(\xi)$ is not known and must be constructed from the known gridcell averages $\bar{\psi}\left[\xi^{(s)}\right]$. This process is referred to as subgrid-cell reconstruction. The accuracy and efficiency of the one-dimensional remapping scheme depends on the subgrid-scale reconstruction method that is usually represented by polynomial functions. Here we use the reconstructions described in Zerroukat et al. (2005) and Zerroukat et al. (2006) where cubic (and lower order) polynomials and parabolic splines are used, respectively. The coefficients $\left[a_{k}^{(i)}, i=0,3\right]$ of the cubic polynomial

$$
\psi_{k}(\xi)=a_{k}^{(0)}+a_{k}^{(1)} \xi+a_{k}^{(2)} \xi^{2}+a_{k}^{(3)} \xi^{3},
$$

in the $k$ th grid cell are computed by requiring that

$$
\begin{aligned}
\int_{\xi_{k}}^{\xi_{k+1}} \psi_{k}(\xi) d \xi & =\bar{\psi}_{k}(\xi), \\
\psi_{k}\left(\xi_{k}\right) & =\psi_{k}^{L}(\xi), \\
\psi_{k}\left(\xi_{k+1}\right) & =\psi_{k}^{R}(\xi), \quad \text { and } \\
\left.\frac{d \psi}{d \xi}\right|_{\left(\xi_{k}+\xi_{k+1}\right) / 2} & =\delta \psi_{k}(\xi),
\end{aligned}
$$

where $\left[\psi_{k}^{L}(\xi), \psi_{k}^{R}(\xi), \delta \psi_{k}(\xi)\right]$ are obtained by a quartic interpolation procedure from the source-gridcell averages (see Zerroukat et al. 2002 for details). The reconstruction (5) is referred to as the piecewise cubic method (PCM). By setting

$$
\delta \psi_{k}(\xi)=\psi_{k}^{R}(\xi)-\psi_{k}^{L}(\xi),
$$

(5) reduces to a quadratic reconstruction referred to as the piecewise parabolic method (PPM) introduced by Colella and Woodward (1984). Furthermore, by setting

$$
\begin{aligned}
& \psi_{k}^{R}(\xi)-\psi_{k}^{L}(\xi)=\delta \psi_{k}(\xi) \quad \text { and } \\
& \psi_{k}^{R}(\xi)+\psi_{k}^{L}(\xi)=2 \bar{\psi}_{k}(\xi)
\end{aligned}
$$

a piecewise linear (PLM) reconstruction results. Finally, by setting

$$
\begin{aligned}
\delta \psi_{k}(\xi) & =0 \quad \text { and } \\
\psi_{k}^{R}(\xi) & =\psi_{k}^{L}(\xi)=\bar{\psi}_{k}(\xi),
\end{aligned}
$$

a piecewise constant ( $\mathrm{PCoM})$ subgrid-scale distribution results. These reconstructions as well as the parabolic spline method (PSM) are used in the current implementation of the algorithm but in general any reconstruction fulfilling (6) can be used. 


\section{c. Monotonic filtering}

An important advantage of the cascade method is the ease at which filters can be applied since they only have to be one-dimensional. Filters do, in general, tend to make the remapping more diffusive (e.g., Lauritzen 2007). It is important that "physical" extrema in the subgrid-cell reconstruction are retained while unphysical ones are filtered out. Less sophisticated filters reduce the subgrid-cell reconstruction to first order for any local extrema, which can be detrimental to accuracy in idealized advection test cases (see, e.g., Table 2 in Nair and Machenhauer 2002). Zerroukat et al. (2005, 2006) refined the filters of Sun et al. (1996) and Nair et al. (1999) and adapted them to the higher-order subgrid-cell reconstructions used here. These filters recursively reduce the order of the subgrid-cell reconstruction until the subgrid-cell distribution is monotone. A nice feature of this filter is that it retains physically allowable extrema instead of reducing the reconstruction to PCoM whenever the local polynomial exhibits so-called monotonicity-violating behavior. Note that this is a relaxed way of implementing monotone filters since small-amplitude over- and undershoots can occur if they are physically allowable. In idealized advection test cases using a cascade transport scheme these filters have been demonstrated to have relatively little impact on the error measures (Zerroukat et al. 2007). For some test cases the accuracy is even improved when applying the filter.

\section{d. The two-dimensional cascade remapping}

To illustrate the cascade remapping in simple terms, we consider a rectangular region on a Cartesian plane overlaid by the source and target grids as depicted in Fig. 2. The idea of the cascade remapping process is closely related to that of the cascade interpolation procedure introduced in Purser and Leslie (1991). Therefore we first describe the cascade interpolation procedure with the aid of Fig. 2.

Let $\left(x^{s}, y^{s}\right)$ and $\left(x^{t}, y^{t}\right)$ be the source and target grids that are represented by dashed lines and solid curves in Fig. 2. Now our task is to transfer data from the source grid points (open circles) to the target grid points (filled circles) as shown in Fig. 2. This is achieved by a "cascade" of one-dimensional interpolation operations from source to target grid through an intermediate-grid system. The intermediate grid is a hybrid grid consisting of intersections of target-grid $x$ lines with source-grid $y$ lines or vice versa. For the present application we consider the intermediate grid as the intersection of targetgrid $x^{t}$ curves with source-grid $y^{s}$ lines (" $\times$ " marks in Fig. 2). Thus the intermediate grid may be expressed as

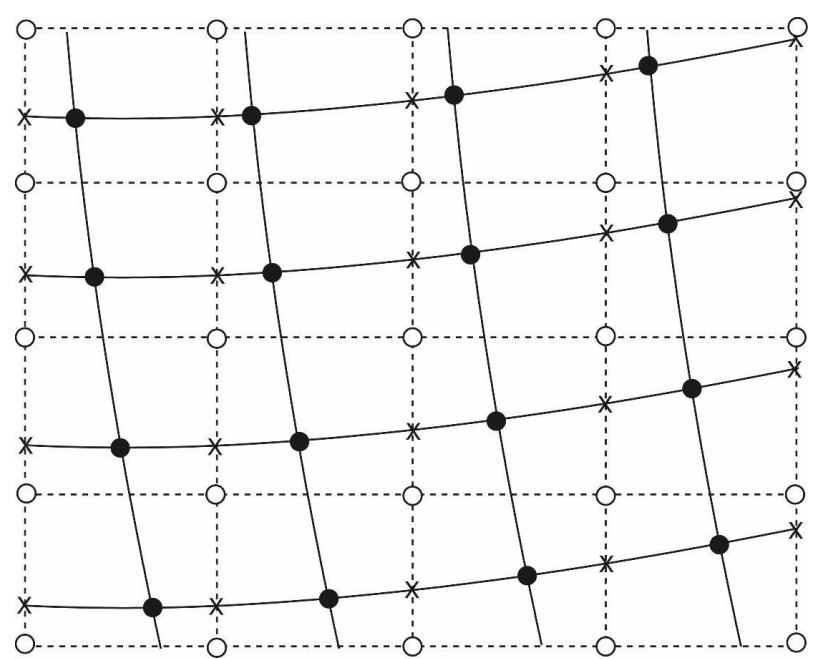

FIG. 2. Schematic illustration of a two-dimensional cascade interpolation from the source grid to the target grid. The source grid $\left(x^{s}, y^{s}\right)$ is represented by dashed lines with open circles as grid points, and the target grid $\left(x^{t}, y^{t}\right)$ is represented by solid curves with filled circles as grid points. The intermediate grid intersections are defined as the intersection of source-grid $y^{s}$ lines with target-grid $x^{t}$ curves and are marked by cross marks $(\times)$.

$\left(x^{\prime}, y^{\prime}\right)$, where $x^{\prime}=x^{s}$ and $y^{\prime}$ is the interpolated or, as done in this article, exactly computed $y$-coordinate values along the target-grid $x^{t}$ curves. The details of intermediate-grid generation and its properties are discussed in Nair et al. (1999).

The first stage (sweep) of cascade interpolation transfers data from source to intermediate grid by means of one-dimensional operations. This is followed by the second stage of one-dimensional interpolations to transfer data from the intermediate to the destination grid, which completes the cascade cycle. The twodimensional conservative remapping can be done in an analogous manner (Nair et al. 2002) where the onedimensional interpolations are replaced by onedimensional remapping operations as described in the previous section. Because of the conservation constraints, the remapping is a more restrictive operation than interpolation. Moreover, as a consistency requirement, the total length (area) of the source, intermediate, and target grids should be the same when conservative cascade remapping is performed. Also, since mass in grid cells is represented by length intervals one should either define the length scale in terms of the actual area of the cells (as done herein) or scale the densities appropriately so that mass is conserved. The approach taken herein guarantees that a constant field is preserved.

Even though the remapping between RLL and cubed-sphere grids, and semi-Lagrangian advection based on the conservative cascade scheme share many 


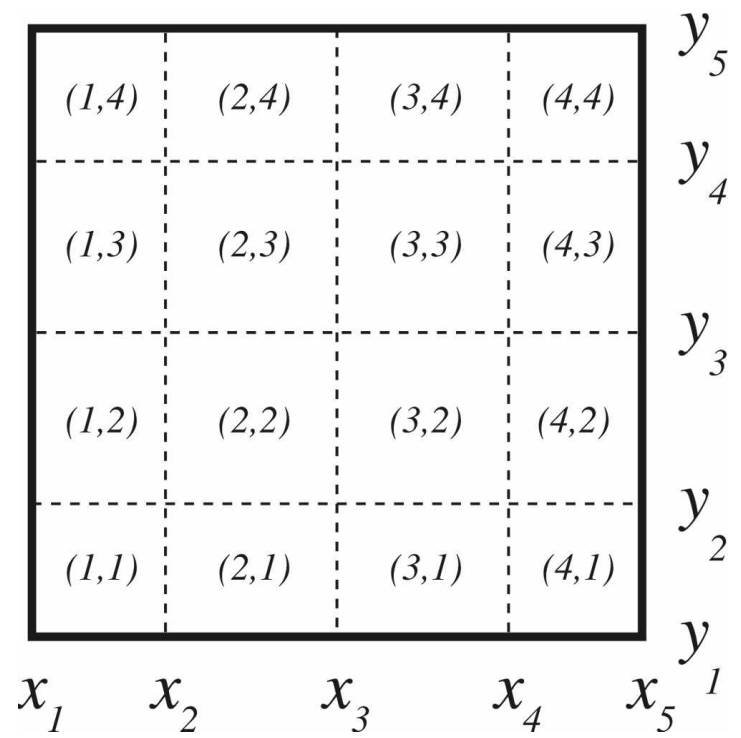

FIG. 3. Schematic illustration of the cubed-sphere grid on the local Cartesian coordinate system centered on each panel as well as the traditional indexing $(i, j)$ used to refer to regular cubedsphere grid cells. The panel index $p$ is omitted since the figure is panel independent.

common features, there are some major differences too. The remapping between RLL and cubed-sphere grids are not connected by "trajectories"; in other words, there is no one-to-one correspondence between the grid points for these two different grid systems. In some sense the remapping between the RLL and cubed sphere is more relaxed. The grid points on the target grid system are dependent only on the neighboring grid points of the source grid and one has more liberty in choosing the intermediate grid and cascade sweep directions. We describe the remapping algorithm between the RLL and cubed sphere in the following sections.

\section{The CaRS method}

The cubed-sphere grid is constructed by the decomposition of the sphere into six identical regions (referred to as panels), obtained by projecting the sides of a inscribed cube onto the sphere. Here we consider the equiangular cubed-sphere mesh so the grid lines on each panel are great-circle arcs. Figure 1 shows the RLL grid on the gnomonic projection and the indexing of the cubed-sphere panels used here. Note that the RLL grid longitudes and equator, which are great-circle arcs, appear as straight lines on a gnomonic projection.

Traditionally the grid lines of the cubed sphere are defined panelwise by employing a local Cartesian grid system. By this definition three indices $(i, j, p)$ are needed to represent the coordinate lines, where $i, j$, and $p$ are the row, column, and panel indices, respectively

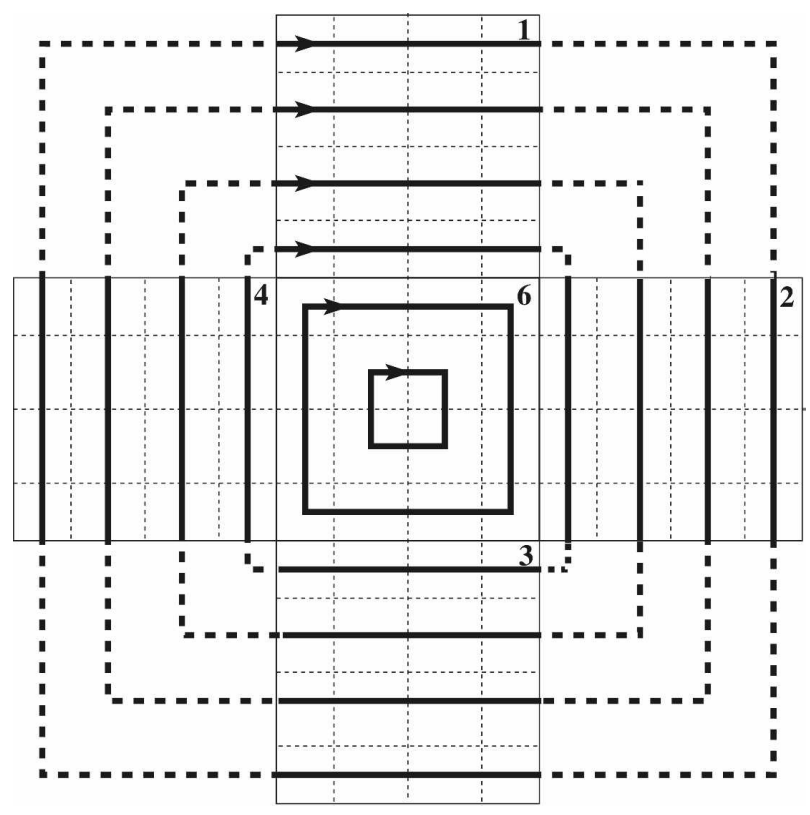

FIG. 4. A schematic illustration of the cubed-sphere latitudes (thick lines with arrows) as projected on a polar tangent plane. These latitudes are logical squares centered at the polar axis of the sphere. The thin dashed lines show the cubed-sphere grid lines on the polar panel 6 and the adjacent equatorial panels (1-4). See also Fig. 5.

(see Fig. 3). For easy implementation of the cascade method we reconstruct the entire cubed-sphere grid system with a family of horizontal and vertical grid lines similar to the longitudes and latitudes of the RLL grid. Also we define the term latitude belt for a collection of contiguous areas (lengths) along a latitude line, and similarly for the longitude belt. We define the cubedsphere latitudes as vertically stacked closed curves (squared patterns) centered about the vertical axis of the sphere as shown on Fig. 4. The cubed-sphere latitude lines are only $C^{0}$ continuous at the four corners. The cubed-sphere latitude belts are intersected by a set of cubed-sphere longitudes. On the equatorial panels each cubed-sphere latitude belt has the same number of intersections (cubed-sphere longitudes). But for the polar panels the number of cubed-sphere longitude intersections decreases from the side to the center. In this interpretation the cubed-sphere grid may be considered to be a deformed reduced RLL grid.

To start with, we consider the remapping from the RLL grid to the cubed-sphere grid. The cascade directions are chosen as follows. The first cascade sweep is in the north-south direction along longitude belts of the RLL grid. For this cascade sweep the source grid is defined in terms of the RLL longitude belt and the target grid is an intermediate grid. Analogous to the two-dimensional Cartesian case, the intermediate grid 
is defined in terms of RLL longitudes and the cubedsphere latitudes. The second cascade sweep is from the intermediate grid to the cubed-sphere latitudes.

The one-dimensional grids used in the cascade cycles are defined in terms of exact areas of the grid cells, leading to what we refer to as an area-consistent approach. For example, for the first cascade sweep the spherical areas of the RLL grid cells along a longitude belt are logically mapped onto a one-dimensional grid [the source grid $\xi^{(s)}$ ], so that there is a one-to-one correspondence between the lengths on $\xi^{(s)}$ and the actual RLL gridcell spherical areas. Similarly for the intermediate grid the exact spherical area of the overlapping between the RLL longitude belt and the cubed-sphere latitude belts define the one-dimensional grid. This area-consistent definition of the one-dimensional grids used for the remapping obviously fulfills the consistency requirement (1); that is, the length of the source and intermediate grids are equal. The spherical areas of the cubed-sphere grid cells along a cubed-sphere latitude define the target grid. This is explained in detail below.

\section{a. Definition of spherical grids}

Consider a RLL grid on a unit sphere with $N_{\lambda}$ longitudes and $N_{\theta}$ latitudes with grid spacing

$$
\Delta \lambda=\frac{2 \pi}{N_{\lambda}} \text { and } \Delta \theta=\frac{\pi}{N_{\theta}-1},
$$

including the pole point so that there are $N_{\lambda} \times\left(N_{\theta}-1\right)$ cells on the RLL grid. The longitudes and latitudes define the walls of the RLL grid cells. For simplicity it is assumed that the longitudes $\lambda=0, \pi / 2, \pi$, and $3 \pi / 2$ are on the RLL grid so that the remapping coefficients are only required to be computed for a quarter of the sphere. The remapping coefficients for the rest of the domain are obtained by symmetry. The algorithm can easily be generalized to accommodate any longitudes of the RLL grids with or without a pole point (see section 3d).

Let $N_{c}$ be the number of grid lines on each panel of the cubed sphere, such that there are $\left(N_{c}-1\right) \times\left(N_{c}-\right.$ 1) cubed-sphere grid cells on each panel. In the local Cartesian gnomonic (or central) projection the grid lines are defined by (e.g., Rančić et al. 1996)

$$
\left(x_{i}, y_{j}\right)=\left(a \tan \alpha_{i}, a \tan \beta_{j}\right), \quad 1 \leq i, \quad j \leq N_{c},
$$

where $a=1 / \sqrt{3}$ is one-half of the length of the cube inscribed into the unit sphere, and the central angles $\left(\alpha_{i}, \beta_{j}\right)$ are given by

$$
\alpha_{i}=-\frac{\pi}{4}+\frac{\pi(i-1)}{2\left(N_{c}-1\right)} \quad \text { and } \quad \beta_{j}=-\frac{\pi}{4}+\frac{\pi(j-1)}{2\left(N_{c}-1\right)} .
$$

(a)

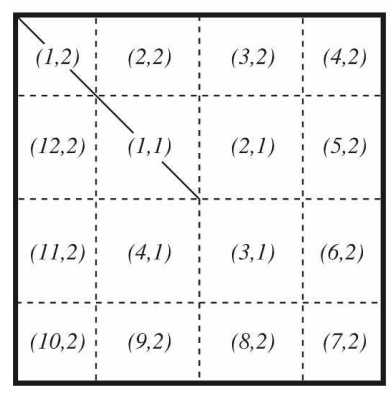

(b)

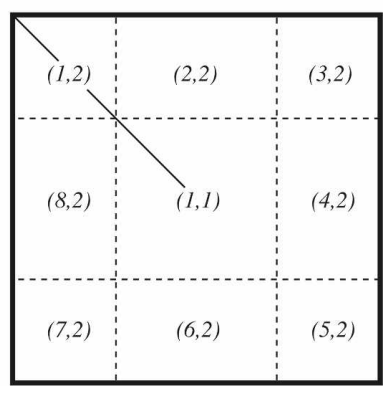

FIG. 5. Schematic illustration of the indexing $(\nu, \ell)$ of the cells along the cubed-sphere latitudes on the South Polar panel (panel 6) for (a) $N_{c}$ odd and (b) $N_{c}$ even. The first index $\nu$ refers to the cell number along the cubed-sphere latitude with index $\ell$. The solid line is $\lambda=0$ longitude and the dashed lines are the cubedsphere grid lines.

The total number of cubed-sphere latitudes in terms of $N_{c}$ is given by

$$
L=2 N_{h}+N_{c}-1 \text {, }
$$

where $N_{h}$ is the integer value of $N_{c} / 2$. Let $(\nu, \ell)$ be the cubed-sphere longitude and latitude indices. As depicted in Fig. 5 the cubed-sphere longitude indexing convention $(\nu)$ is such that the cell in a cubed-sphere latitude belt around or immediately east of $\lambda=0$ has $\nu=1$, and $\nu$ increases eastward. The cubed-sphere latitude indexing is as in the case of RLL grids. The details of the transformation from the traditional $(i, j, p)$ index to cubed-sphere longitude-latitude indexing $(\nu, \ell)$ are given in appendix $\mathrm{B}$.

The RLL grid and the cubed-sphere grid are placed such that the pole points of the RLL grid are located in the center of the cubed-sphere polar panels (panel 5 and 6). Furthermore, the RLL grid is rotated zonally such that the meridian line $(\lambda=0)$ coincides with the edge shared by panel 1 and 4 (see Fig. 6). The more general case is described in section $3 \mathrm{~d}$.

\section{b. The first cascade sweep}

The cascade remapping process consists of three important steps that are one-dimensional remapping from a source to a target grid through an intermediate grid as described in the two-dimensional Cartesian case. In the remapping process the source and target grids are defined in terms of accumulated spherical areas along the direction of the sweeps.

\section{1) SOURCE GRID}

For the first sweep, the source grid is the RLL grid so defining the South Pole as the reference point, $\Lambda_{i 1}^{s}=0$, the discrete accumulated area along a longitude belt $i$, is given by 


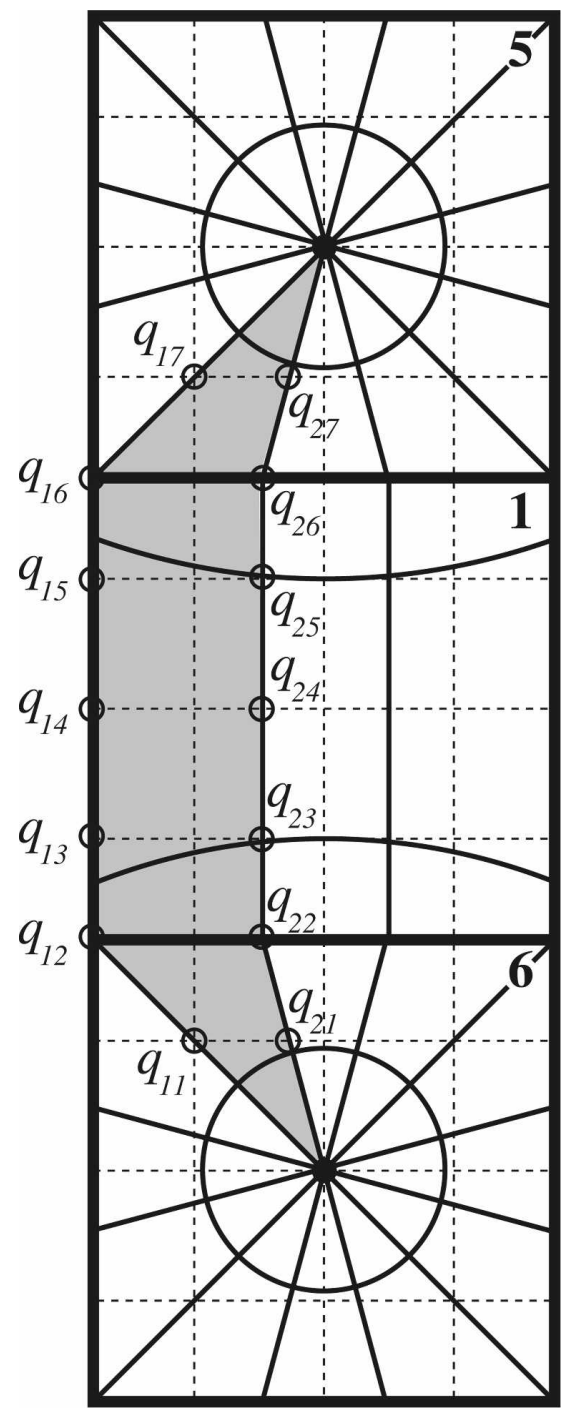

FIG. 6. A schematic illustration of the intermediate grid given the cubed-sphere grid (dashed lines) and regular latitude-longitude grid (thick solid lines) shown in local Cartesian coordinates centered on the surface of the cubed-sphere faces (only one equatorial face is shown). The cubed-sphere face number is in the upper-right corner of each panel. Consider the first, $i=1$, longitudinal belt (shaded area). The open circles encircle the intersections between the cubed-sphere latitudes and the regular longitudes for $i=1,2$. The intersections are denoted $q_{i \ell}$ where the subscripts $i$ and $\ell$ refer to the longitudinal belt and cubed-sphere latitude, respectively. The accumulated areas of the intermediate cells $\Delta_{i \ell}$ along the longitudinal belt $i$ are the areas of the spherical triangles with the South Pole, $q_{i \ell}$, and $q_{(i+1) \ell}$ as edges.

$$
\Lambda_{i j}^{s}=\Delta \lambda\left[\sin \left(\theta_{j}\right)+1\right], \quad j=2, N_{\theta},
$$

for each longitudinal belt $i$. In other words, $\Lambda^{s}$ is the accumulated area along the longitudinal belts. Since an RLL grid is equispaced $\Lambda^{s}$ is identical for all longitudinal belts.

\section{2) INTERMEDIATE GRID}

For the first sweep the intermediate grid is the target grid and is denoted $\Lambda^{t}$. The intermediate grid $\Lambda^{t}$ is defined in terms of the intersections between the RLL grid longitudes and the cubed-sphere latitudes. Consider the part of the sphere located in between longitudes $\lambda=0$ and $\lambda=\pi / 2$ so only panels 1,5 , and 6 are used (see Fig. 6). The intermediate grids on the rest of the sphere are obtained by symmetry. The intersections between the RLL longitudes and cubed-sphere latitudes can easily be computed analytically as described in the following section. Given the location of the intersections in spherical coordinates, the areas of the spherical triangles $\Delta_{i \ell}$ resulting from connecting the South Pole, $q_{i \ell}$, and $q_{(i+1) \ell}$ (see Fig. 6) with great-circle arcs can easily be computed (see appendix A). These spherical triangles are the accumulated areas used to define the intermediate grid along each longitude belt $i$ :

$$
\begin{aligned}
& \quad \Lambda_{i 0}^{t}=0, \\
& \Lambda_{i \ell}^{t}=\Delta_{i \ell}, \quad \ell=1, L-1, \quad \text { and } \\
& \Lambda_{i L}^{t}=4 \pi / N_{\lambda},
\end{aligned}
$$

where $1 \leq i \leq\left(N_{\lambda} / 4\right)-1$. Note that the computation of the spherical areas making up the intermediate grid is exact. The intermediate grid for the rest of the sphere can be computed by symmetry. That is, for $\left(N_{\lambda} / 4\right) \leq$ $i \leq N_{\lambda}$ the intermediate grid $\Lambda^{t}$ is given by

$$
\begin{aligned}
& \quad \Lambda_{i 0}^{t}=0, \\
& \Lambda_{i \ell}^{t}=\Delta_{M \ell}, \quad \ell=1, L-1, \quad \text { and } \\
& \Lambda_{i L}^{t}=4 \pi / N_{\lambda},
\end{aligned}
$$

where $M=\operatorname{MOD}\left(i-1, \mathrm{~N}_{\lambda} / 4\right)+1$ (MOD is the standard FORTRAN modulus function). Note the area consistency in the definition of the grids

$$
\sum_{i=1}^{N_{\lambda}} \Lambda_{i N_{\theta}}^{s}=\sum_{i=1}^{N_{\lambda}} \Lambda_{i L}^{t}=4 \pi .
$$

\section{3) INTERMEDIATE GRID INTERSECTIONS}

By design the gnomonic projection has the useful property that great-circle arcs are images of straight lines on local Cartesian coordinates for each face of the inscribed cube as defined by (16). Thus the intermediate intersections are simply crossings between straight lines on the local Cartesian plane. The straight line segments corresponding to the cubed-sphere latitude defined on $\lambda \in[0, \pi / 2]$ can be defined as

$$
y=y_{\ell} .
$$


The straight line corresponding to the RLL longitude $\lambda_{i}$ on the local Cartesian plane for the South Polar panel $(p=6)$ is given by

$$
y=\tan \left(\frac{3 \pi}{4}-\lambda_{i}\right) x .
$$

Therefore the intersection between (27) and (28) is

$$
(x, y)=\left[\frac{y_{\ell}}{\tan \left(\frac{3 \pi}{4}-\lambda_{i}\right)}, y_{\ell}\right], \quad 1 \leq i \leq N_{\lambda} / 4+1 .
$$

Similarly for the North Polar panel $p=5$. For the equatorial panel $(p=1)$ the RLL longitude is given by

$$
x=a \tan \left(\lambda_{i}-\frac{\pi}{4}\right),
$$

and the corresponding intersection is

$$
(x, y)=\left[a \tan \left(\lambda_{i}-\frac{\pi}{4}\right), y_{\ell}\right], \quad 1 \leq i \leq N_{\lambda} / 4+1 .
$$

These intersections are depicted in Fig. 6. The corresponding intersections in spherical coordinates $(\lambda, \theta)$ are computed using the transformations given in appendix A of Nair et al. (2005) [note that the RLL grid in Nair et al. (2005) is rotated such that the $\lambda=0$ longitude is in the center of panel 1 -the transformation is, however, easily adapted to the current setting where the meridian line coincides with the western edge of panel 1].

\section{c. The second cascade sweep}

After the first cascade sweep the data are transferred to the intermediate grid and in the second cascade sweep they are further transferred to the cubed-sphere cells. For the second cascade sweep $\Theta^{s}$ and $\Theta^{t}$ are the one-dimensional source and target grids, respectively. As described in the previous subsection the intermediate grid is defined in terms of the overlapping spherical areas between the RLL longitudinal belts and the cubed-sphere latitudinal belts. The spherical area of each intermediate grid cell along the RLL longitudinal belts can be computed from $\Lambda^{t}$ :

$$
\Delta_{i \ell}^{\mathrm{int}}=\Lambda_{i(\ell+1)}^{t}-\Lambda_{i \ell}^{t},
$$

where $i$ is the RLL longitude index and $\ell$ is the cubedsphere latitude index. Also in the second cascade sweep, we use the intermediate cell area $\Delta_{i \ell}^{\text {int }}$. The source grid $\Theta^{s}$ is the accumulated spherical area of the intermediate cells along the cubed-sphere latitudes

$$
\Theta_{i \ell}^{s}=\sum_{k=1}^{i} \Delta_{k \ell}^{\mathrm{int}} .
$$

For $\Theta^{t}$ we need the spherical areas of the cubed-sphere cells $\Delta_{i j}^{\text {cube }}$ that are derived in appendix C. As for the other one-dimensional cascade grids, the final target grid is defined in terms of accumulated area along the cascade direction, that is, the accumulated spherical area of the cubed-sphere cells along the cubed-sphere latitudes

$$
\Theta_{\nu \ell}^{t}=\sum_{k=1}^{\nu} \Delta_{k \ell}^{\text {cube }}
$$

where $\Delta^{\text {cube }}$ is referred to in terms of $(\nu, \ell)$ using the transformations given in appendix B.

\section{d. More general cases}

In case the RLL grid is rotated zonally with respect to the cubed-sphere grid-that is, $\lambda=0$ is not an RLL grid line-the algorithm can with minimal modifications be applied. First of all, the longitudes $\lambda=0, \pi$, $\pi / 2,3 \pi / 2$ are added to the RLL grid. In such a situation the definition of $\Lambda^{s}$ given in (19) has to be slightly modified since $\Delta \lambda$ is no longer a constant but varies with longitude, and one must remap mass from the original RLL grid to the refined grid. This is, however, easily done by remapping along the RLL latitude belts from the original source grid to the refined grid (or vice versa). Apart from this modification the algorithm is applied as described above. In fact one can add any multiple of four longitudes $\lambda=\lambda_{i}, \lambda_{i}+\pi / 2, \lambda_{i}+\pi$, and $\lambda_{i}+3 \pi / 2$ to the grid and apply the algorithm as described above.

The remapping algorithm described here is based on a cell-centered finite-volume method. If the data points are on the grid intersections (not the cell-centered averages), for example, as in the standard spectral element method, gridpoint values must be transferred onto a cell-centered grid without violating mass conservation. In a spectral element method the variables are defined at quadrature points. The quadrature points do not have volumes associated with them so it only makes sense to associate volumes to elements and not individual grid points. Therefore one needs to integrate fields to volumes before the application of CaRS. When the data are formulated in terms of cell averages CaRS can be directly applied.

In case of conformal cubic grids (e.g., Rančić et al. 1996; Adcroft et al. 2004) or numerically generated cubed sphere grids, the procedure for finding intermediate intersections may not be viable. Moreover, computing the intermediate areas is impractical. In such cases the intermediate-grid generation and intersec- 
tions could be done as in Nair et al. (2002); however, that is beyond the scope of the present study.

\section{Results}

The new remapping algorithm (CaRS) has been implemented and tested using various analytical fields. For comparison the SCRIP algorithm is used. Note that for the second-order version of SCRIP gradients in spherical coordinates must be specified. In a coupled model setting the gradients can be supplied by the internal numerics of the model components using, for example, spectral methods, finite differences, or finite elements. In the present situation the gradients must be estimated from cell-averaged values. Here we choose to use polynomial subgrid-scale reconstructions for the approximation of the gradients, that is, given the subgrid-scale distribution in the local coordinate system (5), the local gradient in the $k$ th cell is approximated by evaluating $d \psi_{k}(\xi) / d \xi$ in the center of the cell. Performing this operation on the RLL gridcell averages, the gradient components are obviously obtained directly in spherical coordinates. For the cubed sphere the local coordinates used for the gradient approximation are the central angles, $\xi=(\alpha, \beta)$. The gradient in local coordinates must then be transformed to spherical coordinates. This is done using the metric-tensor given in appendix D. Note that although the gradient is approximated with a higher-order reconstruction, the subgridcell variation used in SCRIP is a linear and not a parabola or higher-order polynomial as in the directional sweeps in CaRS. However, it should be noted that in theory SCRIP could be extended for higher-order reconstructions by including more terms in the Taylor expansion [Eq. (7) in Jones 1999] but that approach is not exploited herein.

\section{a. Test cases}

We consider three idealized test cases. Results from the remapping from the RLL grid to the cubed-sphere grid are shown. The reverse remapping was also considered but it did not provide any new insights into the accuracy of the algorithms and hence we only show results from the former. Following Jones (1999) a relatively smooth function resembling a spherical harmonic of order 2 and azimuthal wavenumber 2 (see Fig. 7a),

$$
\psi=Y_{2}^{2}=2+\cos ^{2} \theta \cos (2 \lambda),
$$

and a relatively high frequency wave similar to a spherical harmonic of order 32 and azimuthal wavenumber 16 (see Fig. 7b),

$$
\psi=Y_{32}^{16}=2+\sin ^{16}(2 \theta) \cos (16 \lambda)
$$
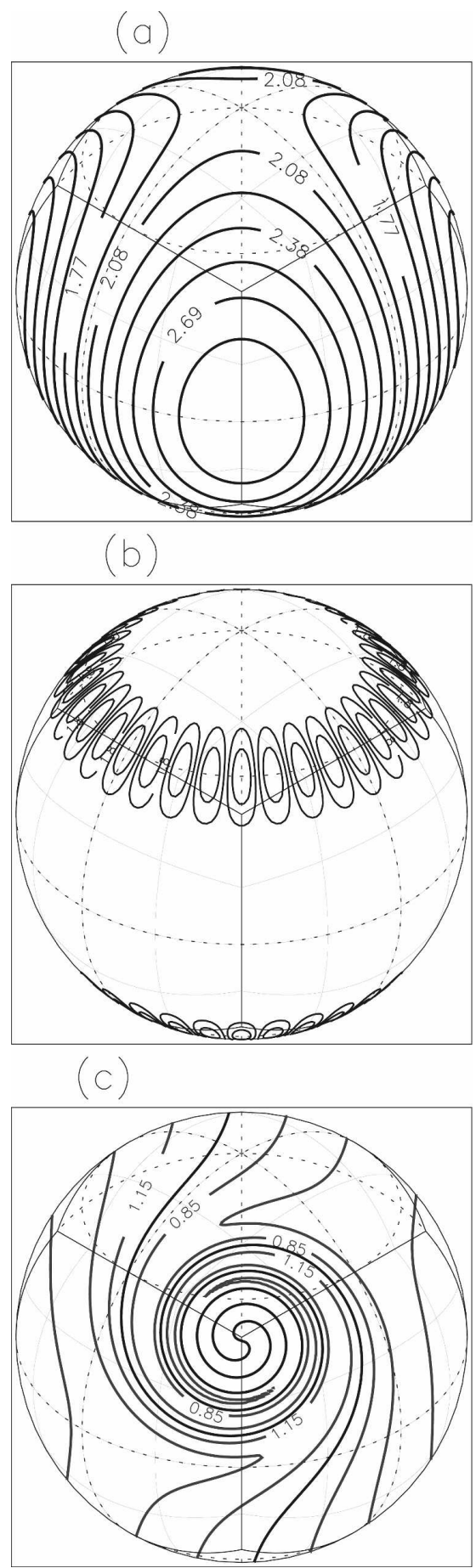

FIG. 7. Contours of the analytical function (a) $Y_{2}^{2}$ at an interval of 0.155 , (b) $Y_{32}^{16}$ at an interval of 0.4 , and (c) the vortex fields with one of the vortices centered about $\left(\lambda_{0}, \theta_{0}\right)=(0,0.6)$ at an interval of 0.15 . Thin lines show the cubed-sphere grid and dashed lines are the regular latitude-longitude grid. 
are used. These two waves test the algorithms performance for a large-scale well-resolved field as well as a higher-frequency wave in the midlatitudes with relatively rapid changing gradients. In addition, a third test case is considered where dual stationary vortex fields (Nair and Machenhauer 2002) are used (see Fig. 7c). The analytic vortex fields are given by

$$
\psi=1-\tanh \left[\frac{\rho^{\prime}}{d} \sin \left(\lambda^{\prime}-\omega^{\prime} t\right)\right],
$$

where the radius $\rho^{\prime}=r_{0} \cos \theta^{\prime}$, angular velocity

$$
\omega^{\prime}\left(\theta^{\prime}\right)=\left\{\begin{array}{ccc}
0 & \text { if } & \rho^{\prime}=0, \\
\frac{V_{t}}{\rho^{\prime}} & \text { if } & \rho^{\prime} \neq 0,
\end{array}\right.
$$

and normalized tangential velocity of the circular vortex is given by $V_{t}=(3 \sqrt{3} / 2) \operatorname{sech}^{2} \rho^{\prime} \tanh \rho^{\prime}$. The $\left(\lambda^{\prime}, \theta^{\prime}\right)$ refer to a rotated spherical coordinate system with the rotated pole located at $\left(\lambda_{0}, \theta_{0}\right)$. For the tests used here we choose $\left(\lambda_{0}, \theta_{0}\right)=(0,0.6), r_{0}=3, d=5$, and $t=6$ (see Fig. 7c), which results in two symmetric vortices located near two diametrically opposite edges of the cube with stronger spatial gradients than $Y_{32}^{16}$ as well as more complex variation over the entire sphere. The vortex centers are slightly off-centered from the vertices of the cubed sphere to avoid possible symmetry with the subdomains. The $Y_{32}^{16}$ and vortex fields have significant variation over the edges of the cubed sphere, thereby challenging the algorithms significantly more that if, for example, fields only had gradients in the equatorial regions.

The performance of the algorithms is quantified using standard error measures:

$$
\begin{gathered}
l_{1} \equiv \frac{I\left(\left|\bar{\psi}_{\text {num }}-\bar{\psi}_{\text {exact }}\right|\right)}{I\left(\bar{\psi}_{\text {exact }}\right)}, \\
l_{2} \equiv \frac{\sqrt{I\left[\left(\bar{\psi}_{\text {num }}-\bar{\psi}_{\text {exact }}\right)^{2}\right]}}{\sqrt{I\left[\left(\bar{\psi}_{\text {exact }} \mid\right)^{2}\right]}}, \\
l_{\infty} \equiv \frac{\max \left(\left|\bar{\psi}_{\text {num }}-\bar{\psi}_{\text {exact }}\right|\right)}{\max \left(\left|\bar{\psi}_{\text {exact }}\right|\right)}, \\
l_{\min } \equiv \frac{\min \left(\bar{\psi}_{\text {num }}\right)-\min \left(\bar{\psi}_{\text {exact }}\right)}{\max \left(\bar{\psi}_{\text {exact }}\right)-\min \left(\bar{\psi}_{\text {exact }}\right)}, \quad \text { and } \\
l_{\max } \equiv \frac{\max \left(\bar{\psi}_{\text {num }}\right)-\max \left(\bar{\psi}_{\text {exact }}\right)}{\max \left(\bar{\psi}_{\text {exact }}\right)-\min \left(\bar{\psi}_{\text {exact }}\right)},
\end{gathered}
$$

where $I()$ is the global integral

$$
I(\bar{\psi})=\sum_{i, j} \bar{\psi}_{i j} \Delta A_{i j}
$$

and $\bar{\psi}_{i j}$ is the average density over the grid cell with area $A_{i j}$. The numerically generated "exact" solution

$$
\bar{\psi}_{\text {exact }}=\frac{1}{\Delta A} \int_{\Delta A} \psi d A
$$

is computed by fourth-order Gaussian quadrature.

\section{b. Discussion of errors}

The errors introduced by the remapping algorithms can be categorized into two types of errors: first, the geometrical approximation to the cells in the remapping algorithm, referred to as the geometrical error, and, second, the approximation to the subgrid-cell distribution, referred to as the derivative error. Of course these errors are to some extent interrelated in the sense that in areas with large geometric error one can also expect increased derivative errors. The derivative error can be reduced by using higher-order subgrid-cell reconstructions; at least in areas with low geometric error. The geometric error can be reduced by increasing the resolution in parts of the domain where the algorithm makes poor approximations to the cells.

The CaRS algorithm splits the two-dimensional remapping problem into two one-dimensional sweeps. Obviously the geometric order reduction of the problem involves approximations that can lead to geometric error and consequently also derivative error. The geometric error in CaRS is expected to be largest near the edges of the cubed-sphere grid faces and near the discontinuities in the derivative of the cubed-sphere latitudes $(\lambda=0, \pi / 2, \pi$, and $3 \pi / 2)$ where the geometrical reduction to one-dimension is least accurate. These angles for which CaRS performs strong stretching are referred to as right angles. The geometric error can be reduced in two ways. First, by construction of the algorithm in terms of the area-consistent definition of the one-dimensional grids used in the remapping. Second, the resolution can be increased in areas where CaRS makes less accurate approximation to the cells.

Depending on the resolution of the source and target grids, and what the source and target grids are, the geometric error can be reduced by optionally adding longitudes to the RLL grid near the right angles (corners) and/or increasing the resolution over the polar cubed-sphere panels, where the approximation to the cells in the directional sweeps of CaRS are least accurate. For example, when remapping from a low-resolution RLL grid to a high-resolution cubed-sphere grid, the geometric error can be reduced significantly by add- 
(a)

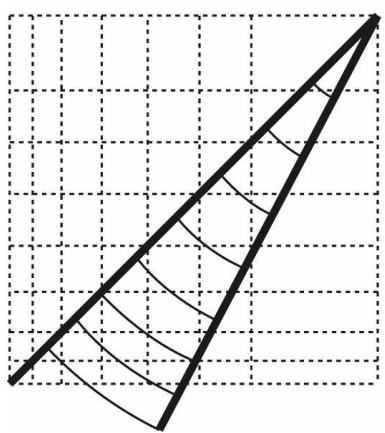

(b)

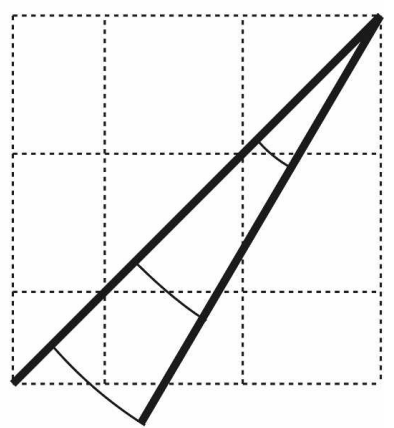

FIG. 8. For the first cascade sweep from an RLL grid to a cubed-sphere grid (dashed lines), the intermediate grid on the polar panels have large geometric error as illustrated schematically on this figure. The intermediate grid is defined in terms of the spherical areas of the intersections between the RLL longitudes (thick solid lines) and the cubed-sphere latitudes (see also Fig. 6). Since the remapping is reduced to a one-dimensional problem the intermediate-grid-cell walls for the first cascade sweep are effectively the latitude segment lines shown in the figure (curved thin solid lines). (a) The situation in which the RLL grid has a coarse resolution compared to the cubed-sphere grid. Near the right angles the effective intermediate cells are a poor approximation to the spherical triangle defining the "exact" intermediate-grid cell. By adding extra longitudes on the RLL grid near the right angles this error can be significantly reduced. (b) The opposite situation in which an increase in the cubed-sphere polar panel resolution significantly reduces the geometrical error.

ing extra longitudes to the RLL grid near the right angles (see Fig. 8a). Similarly when remapping from a high-resolution RLL grid to a low-resolution cubedsphere grid, increasing the resolution on the cubedsphere polar panels can significantly reduce the geometric error (Fig. 8b). On the equatorial panels the geometric error is small since the source and target gridcell walls are aligned in the vertical and are "quasi aligned" in the horizontal (see Fig. 1).

SCRIP uses a fully two-dimensional approximation to the cells by connecting the cell vertices with straight lines in $(\lambda, \theta)$ space. So the approximation to the RLL cells is exact whereas the approximation to the cubedsphere cells is not exact. For example, the approximations to the cell walls made by SCRIP for the south polar panel are not exact since the exact cubed-sphere cell walls are great-circle arcs (see Fig. 9). As for CaRS the approximation to the grid cells is less accurate over the polar panels compared to the equatorial panels.

For the grid pair considered here the anisotropic nature of the RLL grid leads to excessive resolution near the poles relative to the cubed-sphere grid, which has a more uniform resolution over the entire sphere. Hence there can be a loss of information near the poles when remapping back and forth between the cubed-sphere

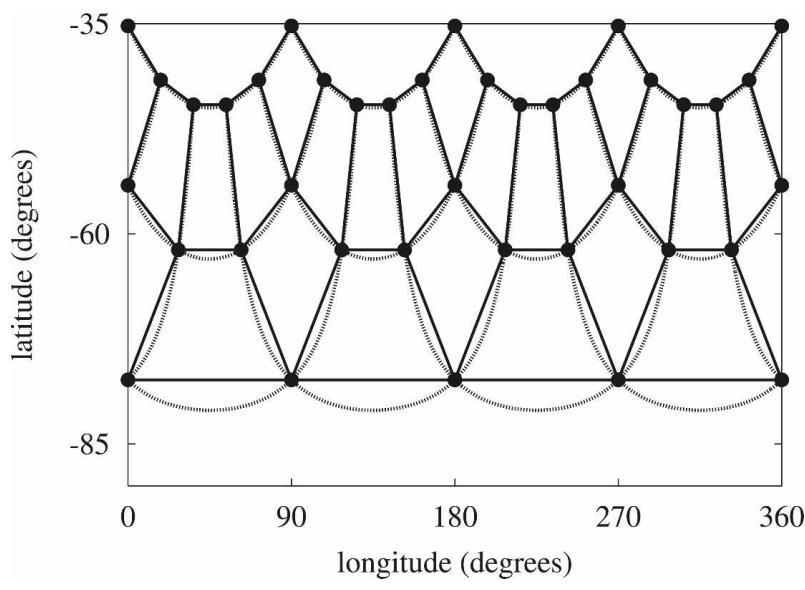

FIG. 9. The cell walls of the cubed-sphere South Polar panel plotted in $(\lambda, \theta)$ space. SCRIP approximates the cells by connecting the cell vertices (filled circles) with straight lines in $(\lambda, \theta)$ space (solid lines). The dotted lines are the exact cell walls that are great-circle arcs.

and RLL grids when these grids have similar resolution in the tropics and consequently have very different resolutions over the poles.

\section{1) Geometric error in CARS}

Remapping from a fine to a coarse grid should basically correspond to an averaging operation, in other words, the derivative error is expected to be small since all the gradient information is lost. Consequently CaRS and SCRIP should yield similar results if their geometric errors are similar. Consider the remapping of $Y_{2}^{2}$ from a fine RLL grid $\left(N_{\lambda}=512, N_{\theta}=256\right)$ to a coarse cubed-sphere grid with resolution $N_{c}=22$. SCRIP based on PCoM, PLM, and PPM for the gradient approximation produces $l_{2}=3.5843 \times 10^{-8}, l_{2}=3.4636 \times$ $10^{-8}$, and $l_{2}=3.4635 \times 10^{-8}$, respectively, which demonstrates that the gradient approximation matters little for this grid pair. Similarly for CaRS we get $l_{2}=4.6241$ $\times 10^{-7}, l_{2}=4.5213 \times 10^{-7}$, and $l_{2}=4.5215 \times 10^{-7}$. The error measure for CaRS is, however, significantly larger than for SCRIP due to the larger geometric error in CaRS. As expected the errors are localized about the right angles (see Fig. 10) as conceptually illustrated in Fig. 8b. The geometric error can be significantly reduced by increasing the cubed-sphere grid resolution on the polar panels. Here we simply double the resolution on the cubed-sphere polar panels for the remapping sweeps and then average to the coarse original cubed-sphere grid after having completed the cascade procedure.

The increase in computational cost for this procedure is approximately $30 \%$ but could be reduced by only increasing resolution near the right angles on the 
(a)

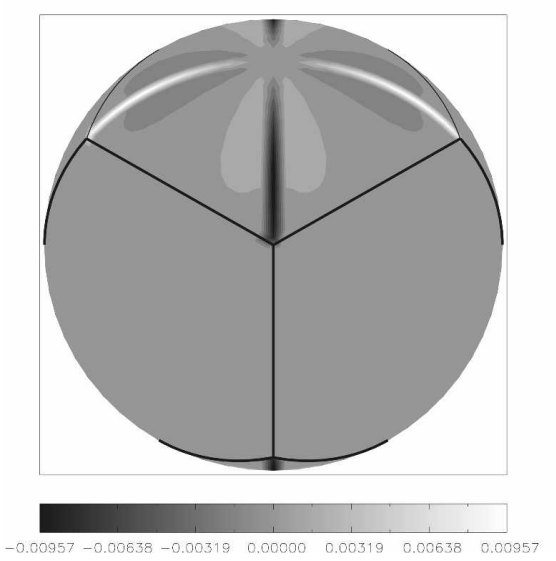

(b)

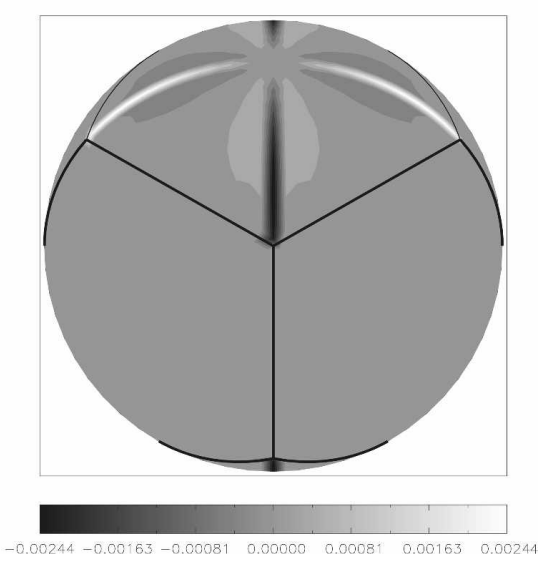

FIG. 10. Contours of the difference between $\bar{\psi}_{\text {exact }}=Y_{2}^{2}$ and the remapped field $\bar{\psi}_{\text {num }}$ for CaRS based on PCM with (a) no refinement and (b) extra longitudes added to the RLL grid as described in the caption of Table 3 .

cubed-sphere polar panels. For this setup with double polar resolution the remapping of $Y_{2}^{2}$ produces $l_{2}=$ $3.2125 \times 10^{-8}, l_{2}=2.8292 \times 10^{-8}, l_{2}=2.8317 \times 10^{-8}$ for reconstructions based on PCoM, PLM, and PPM, respectively. The SCRIP geometric error may be due to the fact that the cells are approximated with straight lines in $(\lambda, \theta)$ space (Fig. 9) that for this particular test setup produces larger errors than the version of CaRS with increased polar panel resolution. Note that one could also apply double resolution in SCRIP over the cubed-sphere polar panels, but the current scope is to use the fully two-dimensional SCRIP as a baseline for reducing the geometric error in CaRS to an acceptable level. Similarly if one is remapping from a fine cubedsphere grid to a coarse RLL grid the geometric error in CaRS can be significantly reduced by adding longitudes about the right angles (not shown). Again the refinement does not lead to major algorithmic changes since they fit directly into the CaRS cascade framework.

\section{2) ACCURACY OF HIGHER-ORDER VERSIONS OF CARS}

An advantage of CaRS is that with modest increase in computational cost one can use high-order subgridcell reconstructions as well as easily incorporate advanced monotone filters. When remapping from a fine to a coarse grid the higher-order reconstructions do not significantly improve accuracy, as shown in the previous section, but when remapping from a coarse to a fine grid the benefit of high-order polynomial reconstructions in CaRS is evident. Here we show results of remapping analytic fields from a coarse RLL grid $\left(N_{\lambda}=\right.$ $\left.128, N_{\theta}=64\right)$ to a fine cubed-sphere grid $\left(N_{c}=130\right)$.
To reduce the errors near the right angles conceptually illustrated in Fig. 8a, we introduce additional longitudes $\pm 1.5(2 \pi / 360)+m \pi / 2$ and $\pm 0.75(2 \pi / 360)+m \pi / 2$, where $m=0,1,2,3$, to the RLL grid. This reduces $l_{2}$ errors by more than a factor 2 (not shown) with an approximately $15 \%$ extra computational cost. Doubling the cubed-sphere polar panel resolution further decreases $l_{1}$ and $l_{2}$ by approximately $4 \%$ but approximately reduces $l_{\infty}$ by $50 \%$ and $30 \%$ for the $Y_{2}^{2}$ and $Y_{32}^{16}$ fields, respectively.

Tables 2 and 3 show performance measures for the remapping of $Y_{2}^{2}, Y_{32}^{16}$, and the idealized vortex field using SCRIP and CaRS, respectively. For SCRIP also analytic approximations to the gradient have been used (by analytic we mean the analytic field gradient evaluated in the center of the cell). It is not possible to specify analytic subgrid-cell reconstructions in CaRS because of the dimensional reduction of the problem that renders exact reconstructions in the cascade sweeps ill-defined.

As expected the $l_{1}$ and $l_{2}$ error norms for both SCRIP and CaRS decrease when going from PCoM, PLM, PPM, to PCM. When using a constant (PCoM) and a linear profile (PLM) for the reconstructions SCRIP and CaRS perform similarly. SCRIP-PPM and SCRIPPCM use higher-order estimates to the gradient based on PPM and PCM, respectively, but the reconstruction profile is still linear. CaRS-PPM, CaRS-PSM, and CaRS-PCM, on the other hand, apply second- and third-order polynomial reconstructions in each cascade sweep, which leads to a significant decrease in the $l_{1}$ and $l_{2}$ error measures relative to SCRIP-PPM and SCRIP-PCM (Tables 2 and 3). 
TABLE 2. Performance measures for the remapping of $Y_{2}^{2}, Y_{16}^{32}$ and the idealized vortices $(\mathrm{Vx})$ from a coarse RLL grid $\left(N_{\lambda}=128\right.$, $\left.N_{\theta}=64\right)$ to a fine cubed-sphere grid $\left(N_{c}=130\right)$ using SCRIP based on different subgrid-cell reconstructions for the gradient approximation as well as with exact gradients (ANA).

\begin{tabular}{|c|c|c|c|c|c|c|}
\hline Method & Field & $l_{1}$ & $l_{2}$ & $l_{\infty}$ & $l_{\min }$ & $l_{\max }$ \\
\hline SCRIP-PCoM & $Y_{2}^{2}$ & $4.4915 \times 10^{-3}$ & $3.0460 \times 10^{-5}$ & $9.1806 \times 10^{-3}$ & $8.5362 \times 10^{-4}$ & $-8.5362 \times 10^{-4}$ \\
\hline SCRIP-PLM & $Y_{2}^{2}$ & $4.0751 \times 10^{-4}$ & $3.4447 \times 10^{-7}$ & $1.6630 \times 10^{-3}$ & $1.0869 \times 10^{-4}$ & $4.9535 \times 10^{-4}$ \\
\hline SCRIP-PPM & $Y_{2}^{2}$ & $3.9186 \times 10^{-4}$ & $3.3448 \times 10^{-7}$ & $1.6550 \times 10^{-3}$ & $-4.9684 \times 10^{-5}$ & $4.9684 \times 10^{-5}$ \\
\hline SCRIP-PCM & $Y_{2}^{2}$ & $3.9110 \times 10^{-4}$ & $3.3342 \times 10^{-7}$ & $1.6538 \times 10^{-3}$ & $-5.0046 \times 10^{-5}$ & $5.0046 \times 10^{-5}$ \\
\hline S-ANA & $Y_{2}^{2}$ & $8.0935 \times 10^{-5}$ & $1.0326 \times 10^{-8}$ & $2.9326 \times 10^{-4}$ & $-5.0237 \times 10^{-5}$ & $5.0237 \times 10^{-5}$ \\
\hline SCRIP-PCoM & $Y_{32}^{16}$ & $1.1458 \times 10^{-2}$ & $5.4858 \times 10^{-4}$ & $8.3619 \times 10^{-2}$ & $5.3716 \times 10^{-2}$ & $-5.3502 \times 10^{-2}$ \\
\hline SCRIP-PLM & $Y_{32}^{16}$ & $3.4194 \times 10^{-3}$ & $5.4405 \times 10^{-5}$ & $3.3244 \times 10^{-2}$ & $2.4733 \times 10^{-2}$ & $2.8909 \times 10^{-2}$ \\
\hline SCRIP-PPM & $Y_{32}^{16}$ & $3.0454 \times 10^{-3}$ & $4.4093 \times 10^{-5}$ & $3.0220 \times 10^{-2}$ & $2.5006 \times 10^{-3}$ & $3.9442 \times 10^{-3}$ \\
\hline SCRIP-PCM & $Y_{32}^{16}$ & $2.9155 \times 10^{-3}$ & $4.0242 \times 10^{-5}$ & $2.9202 \times 10^{-2}$ & $1.4938 \times 10^{-3}$ & $5.0718 \times 10^{-3}$ \\
\hline S-ANA & $Y_{32}^{16}$ & $1.7229 \times 10^{-3}$ & $1.3325 \times 10^{-5}$ & $2.0219 \times 10^{-2}$ & $-2.0353 \times 10^{-2}$ & $2.9545 \times 10^{-2}$ \\
\hline SCRIP-PCoM & $\mathrm{Vx}$ & $7.4300 \times 10^{-3}$ & $1.9459 \times 10^{-4}$ & $8.7704 \times 10^{-2}$ & $8.9093 \times 10^{-5}$ & $-8.9093 \times 10^{-5}$ \\
\hline SCRIP-PLM & $V x$ & $1.9038 \times 10^{-3}$ & $3.1889 \times 10^{-5}$ & $6.4287 \times 10^{-2}$ & $4.6033 \times 10^{-5}$ & $1.2135 \times 10^{-4}$ \\
\hline SCRIP-PPM & $\mathrm{Vx}$ & $1.4705 \times 10^{-3}$ & $1.5282 \times 10^{-5}$ & $3.8388 \times 10^{-2}$ & $1.0839 \times 10^{-5}$ & $-1.0839 \times 10^{-5}$ \\
\hline SCRIP-PCM & $V x$ & $1.4116 \times 10^{-3}$ & $1.3470 \times 10^{-5}$ & $3.7308 \times 10^{-2}$ & $1.1183 \times 10^{-5}$ & $-1.1183 \times 10^{-5}$ \\
\hline
\end{tabular}

Of the subgrid-cell reconstructions considered here, the one leading to the most accurate remappings is quadratic splines (PSM), even though PSM is approximately $60 \%$ more efficient than PPM (and even more when comparing to PCM) in terms of total operation count (Zerroukat et al. 2006). The high accuracy of the PSM is due to the fact that it possesses the optimal reconstruction property: of all piecewise parabolic functions, the quadratic spline is optimal in the sense that it minimizes the integrated square curvature for a given set of cell averages.

\section{3) IMPACT ON ACCURACY OF MONOTONE FILTER}

The CaRS algorithm allows for efficient options for monotone filters that can be incorporated with great ease. Each cascade sweep is one-dimensional; there- fore, monotone filters can be easily implemented. Global under- or overshoots can be detected if $l_{\min }$ and $l_{\max }$ are negative and positive, respectively, for fields that are everywhere positive as for the test cases considered here. However, as discussed in section $2 \mathrm{c}$ the filters do allow for under- and overshoot of minor amplitude if they are physical (not spurious) (see Zerroukat et al. 2007). Table 4 shows that all versions of CaRS applying the monotone filter produce positive $l_{\min }$ and negative $l_{\max }$ norms apart from CaRS based on splines that show minor under- and overshoots. However, most of the unfiltered test results shown in Table 3 are also monotone in terms $l_{\min }$ and $l_{\max }$, indicating that the fields do not challenge the algorithm regarding global under- and overshoots. By remapping a Gaussian hill distribution a negative undershoot would be

TABLE 3. As in Table 2 but for CaRS with longitudes $\pm 1.5(2 \pi / 360)+m \pi / 2$ and $\pm 0.75(2 \pi / 360)+m \pi / 2$, where $m=0,1,2,3$, added to the grid as well as double cubed-sphere polar panel resolution.

\begin{tabular}{|c|c|c|c|c|c|c|}
\hline Method & Field & $l_{1}$ & $l_{2}$ & $l_{\infty}$ & $l_{\min }$ & $l_{\max }$ \\
\hline CaRS-PCoM & $Y_{2}^{2}$ & $4.5743 \times 10^{-3}$ & $3.1900 \times 10^{-5}$ & $9.7831 \times 10^{-3}$ & $8.5362 \times 10^{-4}$ & $-8.5362 \times 10^{-4}$ \\
\hline CaRS-PLM & $Y_{2}^{2}$ & $8.1718 \times 10^{-5}$ & $1.4654 \times 10^{-8}$ & $5.2967 \times 10^{-4}$ & $1.1548 \times 10^{-4}$ & $5.0254 \times 10^{-4}$ \\
\hline CaRS-PPM & $Y_{2}^{2}$ & $2.6905 \times 10^{-5}$ & $1.4736 \times 10^{-9}$ & $3.1578 \times 10^{-4}$ & $5.1533 \times 10^{-7}$ & $-5.1533 \times 10^{-7}$ \\
\hline CaRS-PCM & $Y_{2}^{2}$ & $2.7011 \times 10^{-5}$ & $1.4830 \times 10^{-9}$ & $3.1279 \times 10^{-4}$ & $3.7496 \times 10^{-7}$ & $-3.7495 \times 10^{-7}$ \\
\hline CaRS-PSM & $Y_{2}^{2}$ & $2.7026 \times 10^{-5}$ & $1.3803 \times 10^{-9}$ & $2.8937 \times 10^{-4}$ & $5.9434 \times 10^{-8}$ & $-5.9434 \times 10^{-8}$ \\
\hline CaRS-PCoM & $Y_{32}^{16}$ & $1.1508 \times 10^{-2}$ & $5.5686 \times 10^{-4}$ & $8.3624 \times 10^{-2}$ & $5.3716 \times 10^{-2}$ & $-5.3502 \times 10^{-2}$ \\
\hline CaRS-PLM & $Y_{32}^{16}$ & $2.3237 \times 10^{-3}$ & $2.6073 \times 10^{-5}$ & $3.2057 \times 10^{-2}$ & $1.4450 \times 10^{-2}$ & $4.8177 \times 10^{-2}$ \\
\hline CaRS-PPM & $Y_{32}^{16}$ & $5.5509 \times 10^{-4}$ & $1.1953 \times 10^{-6}$ & $3.6339 \times 10^{-3}$ & $4.4407 \times 10^{-3}$ & $-3.9721 \times 10^{-3}$ \\
\hline CaRS-PCM & $Y_{32}^{16}$ & $4.8890 \times 10^{-4}$ & $9.1914 \times 10^{-7}$ & $3.1819 \times 10^{-3}$ & $4.1227 \times 10^{-3}$ & $-3.5096 \times 10^{-3}$ \\
\hline CaRS-PSM & $Y_{32}^{16}$ & $3.7525 \times 10^{-4}$ & $5.9096 \times 10^{-7}$ & $3.1124 \times 10^{-3}$ & $1.0581 \times 10^{-3}$ & $-1.0418 \times 10^{-3}$ \\
\hline CaRS-PCoM & $V_{x}$ & $7.3023 \times 10^{-3}$ & $1.8609 \times 10^{-4}$ & $8.3958 \times 10^{-2}$ & $8.9093 \times 10^{-5}$ & $-8.9093 \times 10^{-5}$ \\
\hline CaRS-PLM & $V x$ & $1.6198 \times 10^{-3}$ & $3.1286 \times 10^{-5}$ & $5.9024 \times 10^{-2}$ & $4.4973 \times 10^{-5}$ & $1.1881 \times 10^{-4}$ \\
\hline CaRS-PPM & $\mathrm{Vx}$ & $7.5292 \times 10^{-4}$ & $9.1247 \times 10^{-6}$ & $2.9363 \times 10^{-2}$ & $3.2974 \times 10^{-7}$ & $-3.2974 \times 10^{-7}$ \\
\hline CaRS-PCM & $V x$ & $7.0393 \times 10^{-4}$ & $8.1716 \times 10^{-6}$ & $2.8681 \times 10^{-2}$ & $2.7105 \times 10^{-7}$ & $-2.7105 \times 10^{-7}$ \\
\hline CaRS-PSM & $V x$ & $4.3646 \times 10^{-4}$ & $3.0533 \times 10^{-6}$ & $1.8144 \times 10^{-2}$ & $-1.1913 \times 10^{-8}$ & $1.1913 \times 10^{-8}$ \\
\hline
\end{tabular}


TABLE 4. As in Table 3 but for the monotone version of CaRS.

\begin{tabular}{ccccccc}
\hline \hline Method & Field & $l_{1}$ & $l_{2}$ & $l_{\infty}$ & $l_{\min }$ \\
\hline CaRS-PLM-M & $Y_{2}^{2}$ & $1.0001 \times 10^{-4}$ & $2.9694 \times 10^{-8}$ & $9.2017 \times 10^{-4}$ & $8.5362 \times 10^{-4}$ & $-8.5362 \times 10^{-4}$ \\
CaRS-PPM-M & $Y_{2}^{2}$ & $2.6905 \times 10^{-5}$ & $1.4736 \times 10^{-9}$ & $3.1578 \times 10^{-4}$ & $5.1533 \times 10^{-7}$ & $-5.1533 \times 10^{-7}$ \\
CaRS-PCM-M & $Y_{2}^{2}$ & $2.7011 \times 10^{-5}$ & $1.4830 \times 10^{-9}$ & $3.1279 \times 10^{-4}$ & $3.7495 \times 10^{-7}$ & $-3.7495 \times 10^{-7}$ \\
CaRS-PSM-M & $Y_{2}^{2}$ & $2.7026 \times 10^{-5}$ & $1.3803 \times 10^{-9}$ & $2.8937 \times 10^{-4}$ & $5.9434 \times 10^{-8}$ & $-5.9434 \times 10^{-8}$ \\
CaRS-PLM-M & $Y_{32}^{16}$ & $4.1661 \times 10^{-3}$ & $1.0015 \times 10^{-4}$ & $4.0610 \times 10^{-2}$ & $5.3716 \times 10^{-2}$ & $-5.3502 \times 10^{-2}$ \\
CaRS-PPM-M & $Y_{32}^{16}$ & $5.5625 \times 10^{-4}$ & $1.1997 \times 10^{-6}$ & $3.6339 \times 10^{-3}$ & $4.4407 \times 10^{-3}$ & $-3.9721 \times 10^{-3}$ \\
CaRS-PCM-M & $Y_{32}^{16}$ & $4.8992 \times 10^{-4}$ & $9.2326 \times 10^{-7}$ & $3.1809 \times 10^{-3}$ & $4.1227 \times 10^{-3}$ & $-3.5096 \times 10^{-3}$ \\
CaRS-PSM-M & $Y_{32}^{16}$ & $3.7711 \times 10^{-4}$ & $5.9458 \times 10^{-7}$ & $3.1124 \times 10^{-3}$ & $1.0581 \times 10^{-3}$ & $-1.0418 \times 10^{-3}$ \\
CaRS-PLM-M & $\mathrm{Vx}$ & $1.8919 \times 10^{-3}$ & $4.3547 \times 10^{-5}$ & $5.8141 \times 10^{-2}$ & $8.9093 \times 10^{-5}$ & $-8.9093 \times 10^{-5}$ \\
CaRS-PPM-M & $\mathrm{Vx}$ & $1.1250 \times 10^{-3}$ & $2.7013 \times 10^{-5}$ & $5.8376 \times 10^{-2}$ & $3.2974 \times 10^{-7}$ & $-3.2974 \times 10^{-7}$ \\
CaRS-PCM-M & $\mathrm{Vx}$ & $1.0915 \times 10^{-3}$ & $2.6514 \times 10^{-5}$ & $5.8373 \times 10^{-2}$ & $2.7105 \times 10^{-7}$ & $-2.7105 \times 10^{-7}$ \\
CaRS-PSM-M & $\mathrm{Vx}$ & $9.8238 \times 10^{-4}$ & $2.5274 \times 10^{-5}$ & $6.0980 \times 10^{-2}$ & $-1.1913 \times 10^{-8}$ & $1.1913 \times 10^{-8}$ \\
\hline
\end{tabular}

spurious. For the remapping of a Gaussian hill distribution from a coarse RLL grid to a fine cubed-sphere grid (resolutions as in Table 4) using monotone versions of CaRS based on PLM, PPM, PCM, and PSM yields $l_{\text {min }}=0$ and the unfiltered versions of CaRS yield spurious negative undershoots since $l_{\min }=-1.3224 \times$ $10^{-3},-1.6931 \times 10^{-3},-1.5479 \times 10^{-3}$, and $-1.3956 \times$ $10^{-3}$, respectively.

In general the accuracy of the high-order monotone version of CaRS in terms of $l_{1}$ and $l_{2}$ is very similar to the unfiltered CaRS algorithm for the smooth fields $Y_{2}^{2}$ and $Y_{32}^{16}$ (see Table 4). As seen in idealized advection tests the $l_{\infty}$ error increases with the application of monotone filters. For the less well resolved and rapidly varying field of the idealized vortex the impact of the monotone filter is more noticeable in terms of $l_{1}, l_{2}$, and $l_{\infty}$. The filter of Zerroukat et al. (2005) was not designed for low-order reconstructions such as PLM; hence CaRS based on PLM use a standard limiter (e.g., van Leer 1977), which significantly affects the accuracy of the solution relative to the advanced filter of Zerroukat et al. (2005).

\section{c. Discussion on computational cost}

The computation of the geometric information needs only to be computed once and can be reused for any additional field that needs to be remapped. However, the geometric (grid) information used in CaRS can also be efficiently precomputed. Basically the geometric information is based on coordinates for crossings between straight lines on the gnomonic projection. Given the one-dimensional source, intermediate, and target grids, the actual remapping can also be optimized. For a nonfiltered remapping using, for example, PCM the weights for the polynomial reconstruction can also be precomputed since they are solely based on the geometric information. The only information that cannot be precomputed is the filter since it is inherently linked to the field that is being remapped.

\section{Summary}

A general modeling environment consists of various model components usually defined on different grid systems. The accurate transfer of state and flux variables between different grids is crucial to the overall accuracy of the model. Conservation properties are especially important for models intended for long runs as well as monotonicity for the remapping of noisy scalars. Here we present a high-order and monotone regridding method for the specific remapping of scalars between the equiangular cubed-sphere and regular latitudelongitude grid. This new remapping scheme is referred to as CaRS (cascade remapping between spherical grids).

CaRS is based on the cascade method where the twodimensional remapping problem is decomposed into two one-dimensional problems. The advantages of such an approach are that one can apply higher-order methods without excessive increase in computational cost and one has the possibility of applying sophisticated monotone filters. Here we use up to fourth-order methods combined with the advanced monotone filter of Zerroukat et al. (2005).

Since the grids are static we take advantage of the fact that one may freely choose the cascade remapping directions. Also, the geometrical (grid) information needed for the remapping, such as the intersections between grid lines of the two grids, can be precomputed and stored. The remapping directions are chosen to be aligned with the longitudinal belts of the regular latitude-longitude grid and along what is referred to as cubed-sphere latitudes. Instead of defining the cubedsphere grid lines panelwise, as is traditionally done, one 
may reconstruct the cubed-sphere grid with two grid lines similar to the longitudes and latitudes of the regular latitude-longitude grid. Choosing the remapping directions like this insures that the deviation from orthogonality between the cascade sweeps is minimized while exploiting the logical ordering of the data on the different grids.

CaRS is validated by remapping smooth and rapidly varying test functions. We compare standard error measures from remapping with the widely used SCRIP software to the results obtained with CaRS. For the remapping from fine to coarse grids the two methods perform similarly since all gradient information is lost in such situations. In the reverse process, that is, when remapping from a coarse to a fine grid, the higher-order subgrid-cell reconstructions in CaRS come into play. In terms of standard error measures CaRS offers a higherorder alternative to SCRIP that can be more accurate than fully two-dimensional approaches even when monotone filters are applied. However, SCRIP is more general than CaRS since it can, in principle, handle any kind of spherical grids. Generalized versions of CaRS will require some kind of structure in the underlying grid systems so that the cascade method can be applied.

We exploit symmetry and the fact that great-circle arcs appear as straight lines on the gnomonic projection. Therefore we consider remapping from and to a classical cubed-sphere grid based on the equiangular central projection and do not explicitly consider con- formal or numerically generated cubic grids for this study. However, our method may be generalized to accommodate such grids. So far we have only considered the remapping of scalar quantities. The remapping of vectors and the extension of $\mathrm{CaRS}$ to other grids will be addressed in the future.

Acknowledgments. The authors thank Dr. Jones (LANL) for help on getting SCRIP running, Dr. Zerroukat (the Met Office) for help on the implementation of the monotone filters, and Dr. Rasch (NCAR) for encouraging this study. The first author is grateful to NCAR's Advanced Study Program and the Climate Modeling Section for providing necessary support for this research. The second author is partially supported by the DOE SciDac program under award DE-FG0204ER63870. The authors thank Dr. Williamson (NCAR) for valuable comments made during internal review and two anonymous reviewers for their helpful suggestions.

\section{APPENDIX A}

\section{Area of Spherical Triangle}

Consider a spherical triangle with one vertex at the South Pole, and the other two vertices located at $\left(\lambda_{1}\right.$, $\left.\theta_{1}\right)$ and $\left(\lambda_{2}, \theta_{2}\right)$ (see Fig. A1). The sides are defined by great-circle arcs intersecting pairwise in the three vertices. The area $\mathcal{A}$ of the spherical triangle is computed by using the formula

$$
\mathcal{A}=4 \tan ^{-1} \sqrt{\tan \left(\frac{s}{2}\right) \tan \left(\frac{s-a}{2}\right) \tan \left(\frac{s-b}{2}\right) \tan \left(\frac{s-c}{2}\right)}
$$

(e.g., Miller 1994). The auxiliary variables $s, a, b$, and $c$ are given by

$$
\begin{aligned}
& s=\frac{1}{2}(a+b+c), \\
a & =\theta_{1}+\frac{\pi}{2}, \\
b= & 2 \sin ^{-1} \sqrt{\operatorname{hav}(a-c)+\sin a+\sin c \operatorname{hav}\left(\lambda_{2}-\lambda_{1}\right)}, \text { and } \\
c= & \theta_{2}+\frac{\pi}{2},
\end{aligned}
$$

where the haversine function is defined by $\operatorname{hav}(x)=(1-\cos x) / 2$.

\section{APPENDIX B}

\section{Index Transformations}

Let $(i, j)$ be conventional indices of the cell on each panel of the cubed sphere where $1 \leq i, j \leq N_{c}$. The lower left cell in the panel has index $(1,1)$, and $i$ is the column index and $j$ is the row index (see Fig. 3). For the formulation of the remapping algorithm we define cubed-sphere latitudes. The latitudinal index of the cubed-sphere latitudes is denoted $\ell$ and the cells along the cubed-sphere latitudes are denoted $\nu$. The $\nu$ index increases eastward along the cubed-sphere latitude (see Fig. 5). If $N_{c}$ is even, the first cubed-sphere latitude is, by convention, the cell located over the pole. The number of cells in the $\ell$ th cubed-sphere latitude located on the South Polar panel (panel 6) is $4+(\ell-1) 8$. By 


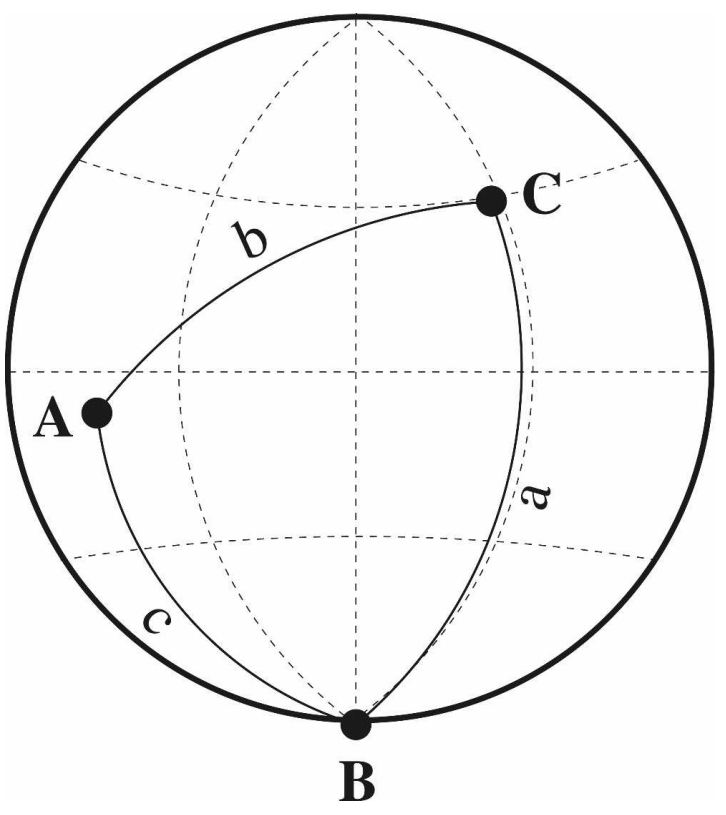

FIG. A1. Spherical triangle with vertices A, B, and C located at $\left(\lambda_{1}, \theta_{1}\right),(0,-\pi / 2)$, and $\left(\lambda_{2}, \theta_{2}\right)$, respectively. The triangle edge lengths are denoted with lowercase letters. Since B is chosen as a vertex at the earth's pole $a$ and $c$ are simply $a=\theta_{1}+\pi / 2$ and $c=$ $\theta_{2}+\pi / 2$. The length of edge $b$ is given by (A4).

symmetry, this is similar for panel 5. For the cubedsphere latitudes on the equatorial panels the number of cubed-sphere latitudes is simply $4\left(N_{c}-1\right)$.

The relationship between the conventional indexing of the cells $(i, j, p)$, where $i$ is the row index, $j$ is the column index, and $p$ is the panel number, and the cubed-sphere latitude indexing in terms of $(\nu, \ell)$ described above is given as follows.

For panel $6\left(1 \leq \ell \leq N_{h}\right)$, the regular row index $i$ is given by

$$
\begin{aligned}
& i=N_{h}-\ell+\nu, \quad 1 \leq \nu \leq e, \\
& i=N_{h}+\ell-1+f, \quad e+1 \leq \nu \leq 2 e, \\
& i=N_{h}+\ell-\nu+f, \quad 2 e+1 \leq \nu \leq 3 e,
\end{aligned}
$$

and

$$
i=N_{h}+1-\nu, \quad 3 e+1 \leq \nu \leq 4 e,
$$

where $N_{h}$ is the integer value of $N_{c} / 2, e$ is one-quarter of the number of cells in a cubed-sphere latitude,

$$
e=1+(\ell-1) 2,
$$

and $f=\operatorname{MOD}\left(N_{c}, 2\right)$ (where MOD is the standard FORTRAN modulus function). The regular column index $j$ is given by

$$
\begin{aligned}
& j=N_{h}+\ell-1+f, \quad 1 \leq \nu \leq e, \\
& j=N_{h}+\ell-\nu+f, \quad e+1 \leq \nu \leq 2 e, \\
& j=N_{h}-\ell+1, \quad 2 e+1 \leq \nu \leq 3 e, \quad \text { and } \\
& j=N_{h}-\ell+\nu, \quad 3 e+1 \leq \nu \leq 4 e .
\end{aligned}
$$

For panels 1-4 $\left(N_{h}+1 \leq \ell \leq N_{h}+N_{c}-1\right)$, the regular row indices $(i, j)$ are given by

$$
\begin{aligned}
& i=\nu+(p-1)\left(N_{c}-1\right) \quad \text { and } \\
& j=\ell-N_{h},
\end{aligned}
$$

where $p$ is the panel number. For panel $5\left(N_{h}+N_{c} \leq\right.$ $\left.\ell \leq 2 N_{h}+N_{c}-1\right)$, by symmetry one can use the formulas for panel 6 but with $\ell$ replaced with $2 N_{h}+$ $N_{c}-\ell$.

\section{APPENDIX C}

\section{Area of Cubed-Sphere Cells}

The cubed-sphere cell is defined by connecting the edges with straight lines in the local Cartesian coordinate system. Since straight lines on the gnomonic projection are great-circle arcs on the sphere, the area of the cubed-sphere cells on the sphere is given by

$$
\Delta^{\text {cube }}=-2 \pi+a_{1}+a_{2}+a_{3}+a_{4},
$$

where $a_{i}$ are the interior spherical angles of the cell. The angles can be expressed in terms of the metric tensor

$$
\cos ^{-1}\left(\frac{g_{12}}{\sqrt{g_{11} g_{22}}}\right)
$$

which for an equiangular projection is given by

$$
\operatorname{ang}(\alpha, \beta)=-\sin \alpha \sin \beta
$$

(see, e.g., Nair et al. 2005), where $(\alpha, \beta)$ are the central angles of the vertex of the cell [see (17)]. The $a_{1}, a_{2}, a_{3}$, and $a_{4}$ in (C1) are given by

$$
\begin{aligned}
& a_{1}=\operatorname{ang}\left(\alpha_{i}, \beta_{j}\right), \\
& a_{2}=\pi-\operatorname{ang}\left(\alpha_{i+1}, \beta_{j}\right), \\
& a_{3}=\pi-\operatorname{ang}\left(\alpha_{i}, \beta_{j+1}\right), \quad \text { and } \\
& a_{4}=\operatorname{ang}\left(\alpha_{i+1}, \beta_{j+1}\right)
\end{aligned}
$$

for the $(i, j)$ th cubed-sphere grid cell.

\section{APPENDIX D}

\section{Rotation of Vectors from Central Angle Cubed-Sphere Coordinates to Regular Latitude-Longitude Coordinates}

Given a vector $\mathbf{v}^{(\alpha, \beta)}=v_{1} \hat{\alpha}+v_{2} \hat{\beta}$ in local cubedsphere central-angle coordinates, the corresponding vector in spherical coordinates is given by 


$$
\mathbf{v}^{(\lambda, \theta)}=\mathbf{Q} \cdot \mathbf{v}^{(\alpha, \beta)},
$$

where the matrix $\mathbf{Q}$ is given by

$$
\begin{gathered}
\frac{\sec \theta \sec \lambda}{R}\left(\begin{array}{cc}
\sec \lambda \cos ^{2} \alpha & \tan \theta \tan \lambda \cos ^{2} \beta \\
0 & \sec \theta \cos ^{2} \beta
\end{array}\right), \\
\frac{1}{R \sin ^{2} \theta}\left(\begin{array}{cc}
-\cos ^{2} \alpha \sin \theta \cos \lambda & \cos ^{2} \beta \sin \theta \sin \lambda \\
-\sin \lambda \cos ^{2} \alpha & \cos \lambda \cos ^{2} \beta
\end{array}\right),
\end{gathered}
$$

and

$$
\frac{1}{R \sin ^{2} \theta}\left(\begin{array}{cc}
\cos ^{2} \alpha \sin \theta \cos \lambda & \cos ^{2} \beta \sin \theta \sin \lambda \\
-\sin \lambda \cos ^{2} \alpha & \cos \lambda \cos ^{2} \beta
\end{array}\right)
$$

(Nair et al. 2005) for panels $1-4,5$, and 6, respectively; $a=R / \sqrt{3}$ is one-half of the length of the cube inscribed into the sphere with radius $R$.

\section{REFERENCES}

Adcroft, A., J. M. Campin, C. Hill, and J. Marshall, 2004: Implementation of an atmospheric-ocean general circulation model on the expanded spherical cube. Mon. Wea. Rev., 132, 2845-2863.

Baer, F., H. Wang, J. J. Tribbia, and A. Fournier, 2006: Climate modeling with spectral elements. Mon. Wea. Rev., 134, 3610 3624.

Colella, P., and P. R. Woodward, 1984: The piecewise parabolic method (PPM) for gas-dynamical simulations. J. Comput. Phys., 54, 174-201.

Dukowicz, J., 1984: Conservative rezoning (remapping) for general quadrilateral meshes. J. Comput. Phys., 54, 411-424.

Giraldo, F. X., and T. E. Rosmond, 2004: A scalable spectral element Eulerian atmospheric model (SEE-AM) for NWP: Dynamical core tests. Mon. Wea. Rev., 132, 133-153.

Jones, P. W., 1999: First- and second-order conservative remapping schemes for grids in spherical coordinates. Mon. Wea. Rev., 127, 2204-2210.

Lauritzen, P. H., 2007: A stability analysis of finite-volume advection schemes permitting long time steps. Mon. Wea. Rev., 135, 2658-2673.

Miller, R. D., 1994: Computing the area of a spherical polygon. Graphics Gems IV, P. S. Heckbert, Ed., Academic Press Graphics Gems Series, Academic Press Professional, 132137.

Nair, R. D., and B. Machenhauer, 2002: The mass-conservative cell-integrated semi-Lagrangian advection scheme on the sphere. Mon. Wea. Rev., 130, 649-667.

_, J. Côté, and A. Staniforth, 1999: Monotonic cascade inter- polation for semi-Lagrangian advection. Quart. J. Roy. Meteor. Soc., 125, 197-212.

— J. S. Scroggs, and F. H. M. Semazzi, 2002: Efficient conservative global transport schemes for climate and atmospheric chemistry models. Mon. Wea. Rev., 130, 2059-2073.

,-- , and,- 2003 : A forward-trajectory global semiLagrangian transport scheme. J. Comput. Phys., 190, 275294

—, S. J. Thomas, and R. D. Loft, 2005: A discontinuous Galerkin transport scheme on the cubed sphere. Mon. Wea. Rev., 133, 814-828.

Purser, R. J., and L. M. Leslie, 1991: An efficient interpolation procedure for high-order three-dimensional semi-Lagrangian models. Mon. Wea. Rev., 119, 2492-2498.

Rančić, M., 1995: An efficient, conservative, monotonic remapping for semi-Lagrangian transport algorithms. Mon. Wea. Rev., 123, 1213-1217.

, R. J. Purser, and F. Mesinger, 1996: A global shallow-water model using an expanded spherical cube. Quart. J. Roy. Meteor. Soc., 122, 959-982.

Ronchi, C., R. Iacono, and P. S. Paolucci, 1996: The "cubed sphere": A new method for the solution of partial differential equations in spherical geometry. J. Comput. Phys., 124, 93114

Sadourny, R., 1972: Conservative finite-difference approximations of the primitive equations on quasi-uniform spherical grids. Mon. Wea. Rev., 100, 136-144.

Sun, W.-Y., K.-S. Yeh, and R.-Y. Sun, 1996: A simple semiLagrangian scheme for advection equations. Quart. J. Roy. Meteor. Soc., 122, 1211-1226.

Thomas, S. J., and R. D. Loft, 2002: Semi-implicit spectral element model. J. Sci. Comput., 17, 92-108.

van Leer, B., 1977: Towards the ultimate conservative difference scheme. IV: A new approach to numerical convection. $J$. Comput. Phys., 23, 276-299.

Zerroukat, M., N. Wood, and A. Staniforth, 2002: SLICE: A semiLagrangian inherently conserving and efficient scheme for transport problems. Quart. J. Roy. Meteor. Soc., 128, 28012820.

$\longrightarrow,-$, and,- 2005 : A monotonic and positive-definite filter for a semi-Lagrangian inherently conserving and efficient (SLICE) scheme. Quart. J. Roy. Meteor. Soc., 131, 2923-2936.

$\longrightarrow,-$, and — 2006: The parabolic spline method (PSM) for conservative transport problems. Int. J. Numer. Methods Fluids, 51, 1297-1318.

,-- , and -2007 : Application of the parabolic spline method (PSM) to a multi-dimensional conservative semiLagrangian transport scheme (SLICE). J. Comput. Phys., 225, 935-948. 